\title{
Identificación de Células Enteroendocrinas Productoras de Péptido Similar al Glucagón Tipo 1 (GLP-1) en el Intestino de la Alpaca
}

\author{
Identification of Producer Enteroendocrine Cells of Glucagon \\ LiKe-PePTide-1 (GLP-1) In THE INTESTINE OF AlPACA
}

César Hidalgo P. ${ }^{1}$, María Vásquez C. ${ }^{1,3}$, Boris Lira M. ${ }^{1}$, José Rodríguez G. ${ }^{2}$

\section{Resumen}

\begin{abstract}
El presente estudio tuvo por objetivo inmunolocalizar la distribución de las células enteroendocrinas L, productoras del péptido similar al glucagón (GLP-1), en el intestino de alpacas. Se utilizaron 36 crías de alpaca de 0-45 días de edad y adultas, distribuidas en siete grupos etarios. Se tomaron muestras de intestino delgado y grueso que fueron procesados mediante inmunohistoquímica, empleando el anticuerpo monoclonal antiGLP-1. Se contó el número de células positivas por eje cripta-vellosidad de cada porción intestinal en cada animal. En el grupo de recién nacidos (RN), el yeyuno fue la porción que contenía más células L, mientras que en los demás grupos fue en el yeyuno e íleon. Respecto a la ubicación, en el duodeno se aprecia un aumento sostenido de células L a partir de los 8 días. El mayor número de células L en el yeyuno y el íleon se presentó en los grupos etarios de RN y 35-45 días de edad, respectivamente, mientras que en el ciego y en el colon se observó en el grupo de animales adultos. Se concluye que las células L en alpacas se encuentran presentes desde el nacimiento, mayoritariamente en yeyuno e íleon.
\end{abstract}

Palabras clave: GLP-1, intestino, células L, alpaca

\section{Abstract}

The aim of this study was to immunolocalize the distribution of producer enteroendocrine cells of glucagon-like peptide (GLP-1) on the epithelial intestine of alpacas. A total of 36 young (0-45 days old) and adult alpacas were used and distributed in seven age groups. The intestinal samples were processed by immunohistochemistry using an

${ }^{1}$ Laboratorio de Fisiología Animal, Facultad de Medicina Veterinaria, Universidad Nacional Mayor de San Marcos, Lima, Perú

${ }^{2}$ Centro de Investigaciones IVITA, Estación Experimental El Mantaro, Universidad Nacional Mayor de San Marcos, Junín, Perú

${ }^{3}$ Email: evasquezc@gmail.com

Recibido: 4 de octubre de 2014

Aceptado para publicación: 6 de marzo de 2015 
anti-GLP-1 monoclonal antibody. Positive cells were counted in 15 crypt-villus axis of each intestinal portion in each animal. In the group of newborns (NB), the jejunum was the portion containing more L-cells, while other groups are in the jejunum and ileum. Regarding location, the duodenum showed a sustained increase of L-cells from day 8. The largest number of L cells in the jejunum and ileum occurred in the age groups of NB and 35-45 days of age, respectively, while in the cecum and colon was observed in the group of adult animals. It is concluded that L-cells in alpacas are present from birth, mainly in the jejunum and ileum.

Key words: GLP-1, intestine, L cells, alpaca

\section{INTRODUCCIÓN}

Las alpacas son los camélidos domésticos de mayor importancia en la economía de los Andes del Perú. La crianza de estos animales, a veces en asociación con ovinos, constituye el principal medio de utilización productiva de extensas áreas de pastos naturales en las zonas alto andinas, donde no es posible la agricultura y la crianza exitosa de otras especies de animales domésticos. Los camélidos convierten con eficiencia la vegetación nativa de estos ambientes en carne y fibra de alta calidad; además, sus pieles y cueros tienen múltiples usos industriales y artesanales (Iñiguez y Alem, 1996).

Las alpacas, como otras especies animales, especialmente en la etapa de lactación, son susceptibles de sufrir afecciones que les pueden conducir a la muerte de no recibir la cantidad necesaria de energía en su dieta, o a causa de trastornos en el metabolismo de los carbohidratos (Esquerre et al., 1976, 1979). Asimismo, diversos estudios en mamíferos han demostrado que el desarrollo de la mucosa y del sistema inmune intestinal depende del alimento consumido y de su calidad, así como de la implantación de la flora bacteriana, ya que el animal nace con un sistema digestivo estéril (Ogra y Welliver, 2008). La alta mortalidad de la alpaca en su etapa posnatal podría disminuirse si se conociera con detalle el desarrollo funcional del tracto intestinal de las crías, especialmente en la parte absortiva de sustancias como la glucosa, así como de la regulación de la homeostasis energética. Es importante indicar que las patologías que cursan con mortalidad en los primeros días de vida, se observan mayormente en el tracto digestivo (Novoa y Flores, 1991; Ramírez, 1991; Rosadio et al., 2012).

El péptido similar al glucagón tipo 1 (GLP-1, por sus siglas en inglés) tiene como función principal, potenciar la secreción de insulina estimulada por glucosa y mejorar la biosíntesis de insulina a través de la inducción de la expresión del gen de insulina (Drucker et al., 1987; Fehmann y Habener, 1992). También estimula la somatostatina e inhibe la secreción de glucagón (Orskov et al., 1988). La activación del GLP-1R aumenta la masa de células a través de la estimulación de la proliferación y la neogénesis y la inhibición de la apoptosis de las células $\beta$ (Drucker 2003; Li et al., 2003). El GLP-1 ejerce estos efectos a través de un GPCR, un miembro de la familia de receptores glucagón-secretina (Thorens et al., 1993), que se expresa ampliamente en los islotes pancreáticos, cerebro, corazón, riñón y tracto gastrointestinal (Thorens et al., 1993; Campos et al., 1994).

En humanos, las células que presentan inmunoreactividad al GLP-1 han sido detectadas desde el yeyuno proximal hasta el rec- 
to, con el uso de un anticuerpo monoclonal específico para el C-terminal de GLP-1 (Eisselle et al., 1992). Un gran número de células en el intestino del cerdo y del humano se encuentran en el yeyuno distal y en el íleon, mientras que en las ratas, la mayor densidad celular se da en el íleon. La marcación ha sido asociada específicamente a las células enteroendocrinas L de la mucosa intestinal, sobre todo en la parte basal, donde se ha encontrado un aumento continuo de la densidad (células $/ \mathrm{mm}^{2}$ de superficie) de las células inmunorreactivas desde la porción proximal hacia la distal del colon, inclusive determinando un gran número celular en el recto; variando la densidad de las células inmunorreactivas al GLP-1, según las especies en un orden característico: ratas, cerdos y el hombre (Eissele et al., 1992).

Se ha demostrado que los camélidos sudamericanos, a diferencia de otras especies, presentan la habilidad de mantener altas concentraciones de glucosa en sangre desde el nacimiento (Esquerre et al., 1976, 1979); sin embargo, ningún estudio ha podido demostrar su distribución intestinal y su rol en la etapa postnatal. Debido a esto, el presente estudio tuvo por objetivo inmunolocalizar la distribución de las células enteroendocrinas L, productoras del péptido similar al glucagón (GLP-1), una incretina de gran importancia debido a su función reguladora de la glucemia.

\section{Materiales y Métodos}

El estudio se llevó a cabo en comunidades alpaqueras de los departamentos de Puno y Cusco, Perú, a una altitud superior a los 3800 metros sobre el nivel del mar; donde las alpacas eran criadas en similares condiciones de manejo y alimentación. El procesamiento de muestras y resultados se realizó en el Laboratorio de Fisiología Animal de la Facultad de Medicina Veterinaria, Universidad Nacional Mayor de San Marcos, Lima, Perú.
Se utilizaron 36 crías de alpacas Huacaya de 0 (menores a $24 \mathrm{~h}$ de nacidos) a 45 días de edad y alpacas adultas. Los animales se encontraban en aparente buen estado de salud. La edad de los animales se confirmó con los registros zootécnicos proporcionados por los dueños de los animales de las comunidades alpaqueras. Todos los animales fueron considerados de descarte por los comuneros y estaban destinados al consumo humano. El manejo de los animales se realizó de acuerdo a la Guía para el Cuidado y Uso de Animales de Laboratorio (National Academy Press, 1996, Washington, DC, EEUU).

Los animales fueron distribuidos en grupos etarios $(0,1-7,8-15,16-21,27-34,35-45$ días, y adultos). Fueron sacrificados con una sobredosis de solución anestésica de pentobarbital sódico al $6.5 \%$, vía vena yugular. Luego, se incidió y se procedió a tomar muestras de 3 a $5 \mathrm{~cm}$ de longitud de la zona media de duodeno, yeyuno, íleon, ciego y colon, las cuales fueron lavadas con formol tamponado al $10 \%$ en la zona de la luz intestinal. Las muestras fueron guardadas en frascos plásticos conteniendo formol tamponado al $10 \%$ por un mínimo de $48 \mathrm{~h}$, antes de su traslado al laboratorio de la ciudad de Lima.

Las muestras fueron reducidas con ayuda de hojas de afeitar a un tamaño aproximado de $0.5 \mathrm{~cm}$ de longitud, conservando la forma circular del intestino. Se colocaron en cápsulas debidamente identificadas y se lavaron con agua corriente durante seis horas. Una vez procesadas, fueron incluidas en parafina, cortadas a un grosor de $5 \mu \mathrm{m}$ y colocadas en láminas portaobjetos embebidas en Poly-L-lysina (Sigma, EEUU). Las láminas fueron desparafinadas, hidratadas a diferentes grados de alcohol e incubadas en metanol al 3\% $\mathrm{H}_{2} \mathrm{O}_{2}$ a temperatura ambiente con el fin de inhibir la peroxidasa endógena. La recuperación antigénica se realizó por irradiación de microondas a $750 \mathrm{~W}$ en citrato buferado (pH 6.0) por dos veces de $5 \mathrm{~min}$. Las uniones no específicas fueron bloquea- 
das con albúmina sérica bovina (BSA) al 1\% (Santa Cruz Biotechnology, EEUU) por 30 min. Las láminas se incubaron durante toda la noche a $4{ }^{\circ} \mathrm{C}$ con el anticuerpo primario monoclonal de ratón anti-GLP-1 (Santa Cruz Biotechnology, EEUU). Se incubó con el kit 'Sistema de detección para inmunoensayos ABC de ratón' (Santa Cruz Biotechnology, EEUU) siguiendo las indicaciones del fabricante. Como cromógeno se utilizó 3’3 diaminobencidina (DAB) y la coloración de contraste se realizó con hematoxilina de Harris. Como controles positivos se utilizaron el íleon y colon ascendente de ratón y como control negativo se utilizaron las mismas muestras en las cuales se reemplazó el anticuerpo primario por BSA $0.1 \%$.

La marcación específica de las células L productoras de GLP-1 se hizo en el eje cripta vellosidad, las cuales se observaron de color marrón dorado. Se contaron las células positivas en cada muestra, seleccionando 15 ejes cripta-vellosidad al azar, por corte intestinal, para todas las porciones intestinales (Zanuzzi et al., 2010).

Para determinar las diferencias entre los valores del número de células entre las diferentes porciones intestinales y las diferentes edades, se utilizó el análisis de varianza de un factor, con un nivel de confianza del $95 \%$.

\section{Resultados y Discusión}

La presencia de células enteroendocrinas L (productoras de GLP-1) (Fig. 1) fue observada en intestino delgado, ciego y colon ascendente, ubicándose a lo largo del eje cripta vellosidad, así como en las criptas colónicas, con variaciones en número según la porción (Cuadro 1). En el grupo de recién nacidos $(\mathrm{RN})$, el yeyuno es la porción que contiene más células $\mathrm{L}$, mientras que en los demás grupos fue en el yeyuno e íleon.

Respecto a la ubicación, en el duodeno se aprecia un aumento sostenido de células $\mathrm{L}$ a partir de los 8 días. El mayor número de células L en el yeyuno y el îleon se presentó en los grupos etarios de $\mathrm{RN}$ y 35-45 días de edad, respectivamente, mientras que en el ciego y en el colon se observó en el grupo de animales adultos.

Las células L tienen por función censar el ingreso de algunos nutrientes y secretar GLP-1 para mantener la homeostasis energética, directa o indirectamente. La forma directa es a través de su accionar en el centro del apetito, ocasionando la saciedad, disminuyendo la motilidad gástrica; mientras que la forma indirecta es a través de su acción sobre las células beta pancreáticas, estimulando la secreción de insulina e incrementando la proliferación celular (Stoffers et al., 2000; Rolin et al., 2002). Una descompensación de los mecanismos de la homeostasis energética podría devenir en la susceptibilidad del animal a padecer enfermedades.

El GLP-1 tiene como función estimular la secreción de insulina (Bayon et al., 2010), lo cual permite tener reservas energéticas (Cunningham, 2009), que son de gran importancia por las altas demandas energéticas de la cría de alpaca (Cid, 2010). El hallazgo de este tipo celular desde el nacimiento hasta la etapa adulta (Cuadro 1) permite establecer que el rol de la homeostasis energética es regulado también por las células $\mathrm{L}$, como sucede en otras especies (Eissele et al., 1992); sin embargo, los resultados no ha permitido esclarecer los altos niveles de glucosa sanguínea que presentan las alpacas, principalmente en los primeros meses de vida.

Las alpacas en la etapa de lactación obtienen su fuente energética (glucosa) de la leche materna. La ingestión de pasturas ocurre a los pocos días de nacer, pero estos alimentos fibrosos no serán digeridos totalmente hasta que el individuo no tenga un estómago con compartimentos totalmente maduros y con capacidad de convertir los pastos en ácidos grasos volátiles; sin embargo, esta fibra pasará hacia el ciego y colon donde por acción bacteriana se convertirán en ácidos grasos de cadena corta, sustancias con gran 


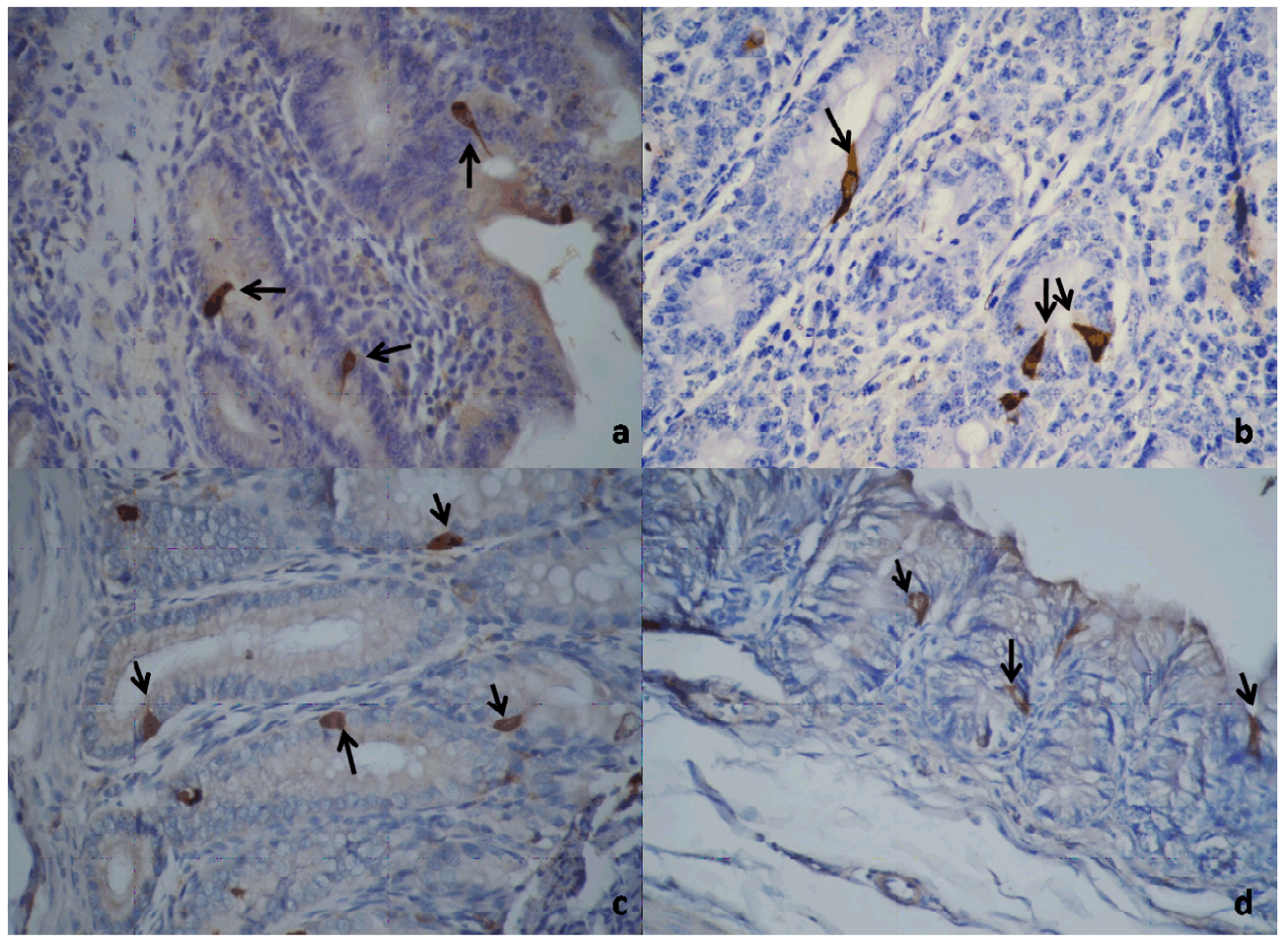

Figura 1. Células L (flechas) identificadas con anticuerpo monoclonal de ratón anti-GLP-1. a) Yeyuno, cría de 21 días de edad, 400x; b) Íleon, cría de 27 días de edad, 400x; c) Colon ascendente, cría de 21 días de edad, 400x; d) Colon ascendente, cría recién nacida, 400x. Coloración de contraste: hematoxilina de Harris

Cuadro 1. Número de células enteroendocrinas L en intestino de alpacas (células L/eje cripta vellosidad), según grupo etario

\begin{tabular}{lcccccc}
\hline $\begin{array}{l}\text { Edad } \\
\text { (días) }\end{array}$ & $\mathrm{n}$ & Duodeno & Yeyuno & Íleon & Ciego & Colon ascendente \\
\hline $\mathrm{RN}$ & 4 & $0.63 \pm 0.71^{\mathrm{a} 1}$ & $1.73 \pm 0.64^{\mathrm{b} 1}$ & $0.67 \pm 0.72^{\mathrm{a} 1}$ & $0.53 \pm 0.64^{\mathrm{a} 1}$ & $0.47 \pm 0.51^{\mathrm{a} 1}$ \\
$1-7$ & 6 & $0.66 \pm 0.56^{\mathrm{a} 1}$ & $1.28 \pm 0.63^{\mathrm{b} 23}$ & $1.23 \pm 0.58^{\mathrm{b} 2}$ & $0.39 \pm 0.49^{\mathrm{c} 1}$ & $0.58 \pm 0.59^{\mathrm{a} 12}$ \\
$8-15$ & 6 & $0.96 \pm 0.45^{\mathrm{a} 23}$ & $1.17 \pm 0.46^{\mathrm{a} 3}$ & $1.71 \pm 0.88^{\mathrm{b} 3}$ & $0.49 \pm 0.59^{\mathrm{c} 1}$ & $0.6 \pm 0.49^{\mathrm{c} 12}$ \\
$16-21$ & 6 & $0.85 \pm 0.48^{\mathrm{a} 12}$ & $1.23 \pm 0.48^{\mathrm{b} 23}$ & $1.34 \pm 0.50^{\mathrm{b} 23}$ & $0.43 \pm 0.54^{\mathrm{c} 1}$ & $0.75 \pm 0.69^{\mathrm{a} 12}$ \\
$27-34$ & 4 & $1.01 \pm 0.45^{\mathrm{a} 23}$ & $1.34 \pm 0.60^{\mathrm{b} 23}$ & $1.45 \pm 0.65^{\mathrm{b} 23}$ & $0.54 \pm 0.57^{\mathrm{c} 1}$ & $0.82 \pm 0.58^{\mathrm{a} 2}$ \\
$35-45$ & 6 & $0.95 \pm 0.45^{\mathrm{a} 23}$ & $1.45 \pm 0.62^{\mathrm{b} 2}$ & $1.75 \pm 0.74^{\mathrm{c} 3}$ & $0.47 \pm 0.57^{\mathrm{d} 1}$ & $0.84 \pm 0.68^{\mathrm{a} 2}$ \\
Adulto & 4 & $1.17 \pm 0.87^{\mathrm{a} 3}$ & $1.24 \pm 0.44^{\mathrm{a} 23}$ & $1.3 \pm 0.47^{\mathrm{a} 2}$ & $0.6 \pm 0.62^{\mathrm{b} 1}$ & $1.2 \pm 0.61^{\mathrm{a} 3}$ \\
\hline
\end{tabular}

$\mathrm{RN}=$ recién nacido y sin ingestión de calostro

${ }^{1,2,3}$ Números diferentes indican diferencia entre edades (columnas) $(p \varangle 0.05$ )

$a, b, c$ Letras diferentes indican diferencia entre porciones intestinales (filas) $(p \varangle 0.05)$ 
capacidad de ser absorbidas en el yeyuno e íleon (donde hay mayor número de células $\mathrm{L}$ de acuerdo al presente estudio) y de allí pasar a circulación sistémica, para almacenarse en el hígado, y convertirse en glucosa cuando el organismo lo requiera. Además, estos ácidos de cadena corta al interactuar con sus receptores en la célula $\mathrm{L}$ intestinal, desencadenan una cascada intracelular que conlleva a la producción y secreción del GLP-1, el cual modula parte del metabolismo energético del individuo (Reimer y McBurney, 1996; Tolhurst et al., 2012).

En el presente estudio se demuestra la presencia de células $L$ en intestino de alpacas desde el nacimiento. En otros estudios se han utilizado las porciones distales del intestino que es donde se encuentran en mayor cantidad, como es el caso del íleon (Ritzel et al., 1997) y colon (Cani et al., 2007) de ratas, en el yeyuno e íleon de cerdos y humanos (Eissele et al., 1992) y en íleon y yeyuno de cuyes lactantes (Anglas, 2012). La ubicación de estas células en porciones distales del intestino delgado o en el colon es debido a su fisiología. Estas células, a nivel del lumen intestinal detectarán la presencia de sustancias como carbohidratos (Margolskee et al., 2007), lípidos y péptidos (Hall et al., 2003) que no han sido absorbidos en las primeras porciones intestinales. Al detectarse estas sustancias se activará a nivel intracelular el cAMP (Rozengurt y Sternini, 2007), el cual desencadenará un proceso que permitirá la salida del calcio del retículo endoplasmático para que este movilice vesículas que contienen GLP-1 y pasen a la sangre.

Asimismo, los transportadores de glucosa SGLT-1 a nivel apical y GLUT-2 a nivel basolateral del enterocito permitirán el ingreso de glucosa, el cual formará ATP, que cerrará canales de $\mathrm{K}$ despolarizando la membrana celular y abriendo los canales de calcio para que ingrese a la célula y permita la secreción de GLP-1. Este sería el mecanismo por el cual en alpacas se da la secreción de GLP-1, ya que estos animales cuentan con transportadores de glucosa desde el nacimiento (Rodríguez et al., 2012). Además de estos mecanismos de secreción, el GLP-1 es secretado por la acción colinérgica de las terminaciones nerviosas del vago, especialmente en las porciones del intestino delgado posterior y el colon (Abello et al., 1994; Balks et $a l .$, 1997), como por ejemplo en el íleon de cerdos (Hansen et al., 2004).

La secreción de GLP-1 en la parte final del intestino delgado y colon, se debe a que el organismo trata de potenciar la homeostasis energética a partir de sustancias no absorbidas en las primeras porciones intestinales (Elliott et al., 1993); además de permitir un mejor trabajo por el páncreas endocrino al estimular la secreción de insulina y el incremento de las células beta por acción del GLP-1 (Stoffers et al., 2000; Rolin et al., 2002).

\section{Conclusiones}

- Las células L en alpacas se encuentran presentes desde el primer día de vida, lo que indicaría su actividad sobre la regulación de la glucemia en esta especie desde el nacimiento.

- El yeyuno e íleon son las porciones intestinales que contienen el mayor número de células L en la alpaca.

\section{Agradecimientos}

$\mathrm{El}$ presente estudio fue financiado por el Proyecto FINCyT No 65 -PIBAP-2008.

\section{Literatura Citada}

1. Abello J, Ye F, Bosshard A, Bernard C, Cuber JC, Chayvialle JA. 1994. Stimulation of glucagon-like peptide-1 secretion by muscarinic agonist in a murine intestinal endocrine cell line. Endocrinology 134: 2011-2017. 
2. Anglas JC, Cueva S, Vásquez M, Lira B, Espinoza J, Lucas J, Rodríguez J. 2012. Identificación y evaluación de transportadores de glucosa SGLT1 y GLUT2 y la incretina Glp-1 en intestino delgado de cuyes (Cavia porcellus). Rev Inv Vet Perú 23: 399-405. doi: 10.15381/rivep.v23i4.932

3. Balks HJ, Holst JJ, von zur Mühlen A, Brabant G. 1997. Rapid oscillations in plasma glucagon-like peptide-1 (GLP-1) in humans: cholinergic control of GLP-1 secretion via muscarinic receptors. J Clin Endocrinol Metab 82: 786-790.

4. Bayón C, Barriga M, Litwak L. 2010. Incretinas, incretinomiméticos, inhibidores de DPP IV (2da parte). Rev Argent Endocrinol Metab 47(3): 39-54.

5. Campos RV, Lee YC, Drucker DJ. 1994. Divergent tissue-specific and developmental expression of receptors for glucagon and glucagon-like peptide1 in the mouse. Endocrinology 134: 21562164. doi: 10.1210/endo.134.5.8156917

6. Cani PD, Holst JJ, Drucker DJ, Delzenne NM, Thorens B, Burcelin R, Knauf C. 2007. GLUT2 and the incretin receptors are involved in glucose-induced incretin secretion. Mol Cell Endocrinol 276: 18-23. doi:10.1016/j.mce. 2007.06.003

7. Cid. 2010. Sanidad de alpacas en la etapa neonatal. En: Manual para estudiantes y profesionales de veterinaria. Madrid, España: Ed Complutense. p 81-82, 144.

8. Cunningham J. 2009. Fisiología respiratoria. $4^{\mathrm{a}}$ ed. Barcelona: Elsevier. 700 p.

9. Drucker DJ, Philippe J, Mojsov S, Chick WL, Habener JF. 1987. Glucagon-like peptide I stimulates insulin gene expression and increases cyclic AMP levels in a rat islet cell line. Proc Natl Acad Sci USA 84: 3434-3438.

10. Drucker DJ. 2003. Glucagon-like peptides: regulators of cell proliferation, differentiation and apoptosis. Mol Endocrinol 17: 161-171. doi: 10.1210/ me.2002-0306
11. Eisselle R, Göke R, Willemer S, Harthus HP, Vermeer H, Arnold R, Göke B. 1992. Glucagon-like peptide-1 in cells in the gastrointestinal tract and pancreas of rat, pig and man. Eur J Clin Invest 22: 283-291.

12. Elliott RM, Morgan LM, Tredger JA, Deacon S, Wright J, Marks V. 1993. Glucagon-like peptide-1 (7-36)amide and glucose-dependent insulinotropic polypeptide secretion in response to nutrient ingestion in man: acute postprandial and 24-h secretion patterns. J Endocrinol 138: 159-166. doi: 10.1677/ joe. 0.1380159

13. Esquerre C, Samaniego L, Román M. 1976. Estadios metabólicos en relación al desarrollo post natal de la alpaca (Lama pacos). En: I Congreso Peruano de Bioquímica. Lima, Perú.

14. Esquerre C, Samaniego L, Navas M, Sánchez D. 1979. Variaciones metabólicas asociadas al crecimiento post natal de la alpaca. En: XII Congreso Peruano de Química. Lima, Perú.

15. Fehmann HC, Habener JF. 1992. Insulinotropic hormone glucagon-like peptide-I(7-37) stimulation of proinsulin gene expression and proinsulin biosynthesis in insulinoma beta TC-1 cells. Endocrinology 130: 159-166. doi: 10.1210/endo.130.1.1309325

16. Hall WL, Millward DJ, Long SJ, Morgan LM. 2003. Casein and whey exert different effects on plasma amino acid profiles, gastrointestinal hormone secretion and appetite. Br J Nutr 89: 239248. doi: 10.1079/BJN2002760

17. Hansen L, Lampert S, Mineo H, Holst J. 2004. Neural regulation of glucagonlike peptide-1 secretion in pigs. Am J Physiol Endocrinol Metab 287: E939-947. doi: 10.1152/ajpendo.00197.2004

18. Iñiguez, LC, Alem R. 1996. Role of camelids as means of transportation and exchange in the Andean region of Bolivia. World Anim Rev 86: 12-21.

19. Li Y, Hansotia T, Yusta B, Ris F, Halban PA, Drucker DJ. 2003. Glucagon-like peptide-1 receptor 
signaling modulates beta cell apoptosis. J Biol Chem 278: 471-478. doi: 10.1074/ jbc.M209423200

20. Margolskee RF, Dyer J, Kokrashvili Z, Salmon KS, Ilegems E, Daly K, Maillet EL, et al. 2007. T1R3 and gustducin in gut sense sugars to regulate expression of $\mathrm{Na}+$-glucose cotransporter 1. Proc Natl Acad Sci USA 104: 1507515080.

21. Novoa C, Flores A. 1991. Producción de rumiantes menores: alpacas. Lima: Rerumen. 358 p.

22. Ogra P, Welliver R. 2008. Effects of early environment on mucosal immunologic homeostasis, subsequent immune responses and disease outcome. Nestlé Nutr Workshop Ser Pediatr Program 60: 145-181. doi: 10.1159/ 0000113492

23. Orskov C, Holst JJ, Nielsen OV. 1988. Effect of truncated glucagon-like peptide-1 [proglucagon- (78-107) amide] on endocrine secretion from pig pancreas, antrum, and nonantral stomach. Endocrinology 123: 2009-2013

24. Ramírez A. 1991. Enfermedades infecciosas. En: Fernández-Baca $S$ (ed). Avances y perspectivas del conocimiento de los camélidos sudamericanos. Santiago de Chile: FAO. p 263-324.

25. Reimer RA, McBurney MI. 1996. Dietary fiber modulates intestinal proglucagon messenger ribonucleic acid and postprandial secretion of glucagonlike peptide- 1 and insulin in rats. Endocrinology 137: 3948-3956. doi: 10.1210/endo.137.9.8756571

26. Ritzel U, Fromme A, Ottleben M, Leonhardt U, Ramadori G. 1997. Release of glucagon-like peptide-1 (GLP1) by carbohydrates in the perfused rat ileum. Acta Diabetol 34: 18-21.

27. Rodríguez J, Cueva S, Lira B, Espinoza J, Vásquez M. 2012. Identificación inmunohistoquímica de transportadores de glucosa intestinal y absorción de glucosa durante el desarrollo y maduración del intestino delgado de crías de alpacas. Rev Inv Vet Perú 23: 126137. doi: $10.15381 /$ rivep.v23i2.892

28. Rolin B, Larsen MO, Gotfredsen CF, Deacon CF, Carr RD, Wilken M, Knudsen LB. 2002. The long-acting GLP-1 derivative NN2211 ameliorates glycemia and increases â-cell mass in diabetic mice. Am J Physiol Endocrinol Metab 283:E745-E752. doi: 10.1152/ ajpendo.00030.2002

29. Rosadio R, Maturrano L, Pérez D, Luna E. 2012. El complejo entérico neonatal en alpacas andinas. Rev Inv Vet Perú 23: 261-271. doi: 10.15381/ rivep.v23i3.907

30. Rozengurt E, Sternini C. 2007. Taste receptor signaling in the mammalian gut. Curr Opin Pharmacol 7: 557-562. doi: 10.1016/j.coph.2007.10.002

31. Stoffers DA, Kieffer TJ, Hussain MA, Drucker DJ, Egan JM, Bonner-Weir S, Habener JF. 2000. Insulinotropic glucagon-like peptide-1 agonists stimulate expression of homeodomain protein IDX1 and increase islet size in mouse pancreas. Diabetes 49: 741-748. doi: 10.2337/diabetes.49.5.741

32. Thorens B, Porret A, Bühler L, Deng SP, Morel P, Widmann C. 1993. Cloning and functional expression of the human islet GLP-1 receptor: demonstration that exendin- 4 is an agonist and exendin-(9-39) an antagonist of the receptor. Diabetes 42: 1678-1682. doi: 10.2337/diab.42.11.1678

33. Tolhurst G, Heffron H, Lam YS, Parker HE, Habib AM, Diakogiannaki E, Cameron J, et al. 2012. Short-chain fatty acids stimulate glucagon-like peptide-1 secretion via the G-protein-coupled receptor FFAR2. Diabetes 61: 364-371. doi: 10.2337/db111019

34. Zanuzzi C. 2010. Efectos de la intoxicación con Solanum glaucophyllum (duraznillo blanco) sobre la proliferación, diferenciación y muerte celular en el intestino de conejos. Tesis Doctoral. Argentina: Universidad Nacional De La Plata. 286 p. 